\title{
Ultraprecise Photometry from Space: The MOST Microsat Mission
}

\author{
J. M. Matthews, R. Kuschnig, G. A. H. Walker, J. Pazder, R. Johnson, \\ K. Skaret, E. Shkolnik, T. Lanting, J. P. Morgan, S. Sidhu \\ Department of Physics \& Astronomy, University of British Columbia, \\ Vancouver, B.C., V6T 1Z\& Canada
}

\begin{abstract}
MOST (Microvariability \& Oscillations of STars / Microvariabilité et Oscillations STellaire) is a Canadian microsatellite mission intended to detect rapid photometric oscillations at the $\mu \mathrm{mag}$ level in stars brighter than $V \sim 6$. This limit is set primarily by the $15-\mathrm{cm}$ aperture of the MOST telescope. The small size and mass of the MOST bus (similar to a suitcase) sets a limit on the pointing accuracy of about \pm 10 arcsec. To achieve the required photometric precision under these conditions, the MOST focal plane features a set of Fabry microlenses which can spread the target starlight into a pupil image of the telescope onto a CCD. The large size ( $\sim 1600$ pixels) and positional stability ( \pm 0.1 pixel) of these images makes MOST insensitive to CCD flat-fielding errors. MOST is currently on schedule to be launched in early 2002 .
\end{abstract}

The goal of asteroseismology is to learn about the internal structure and evolutionary histories of pulsating stars. In particular, if we can obtain high-S/N eigenspectra of p-mode oscillations in other Sun-like stars, we can constrain their masses or radii, and make independent estimates of their ages based on their core compositions. But in the Sun's integrated light, the eigenspectrum has very low amplitudes (a few $\mathrm{cm} \mathrm{s}^{-1}$ in velocity and several $\mu$ mag in light) and frequency splittings of only a few $\times 0.1 \mu \mathrm{Hz}$.

To achieve this sensitivity and resolution (without aliasing), random noise sources at short time scales must be reduced to very low levels, and observations of a given target must span many weeks with a high duty cycle. In ground-based photometry, the dominant noise source at high frequencies is atmospheric scintillation. This can only be suppressed by using very large apertures (which average over many turbulent cells in the telescope beam) or by going into space. The high duty cycle can only be achieved on the ground by a network of dedicated large telescopes, or a single large telescope at one of the poles (impractical because of the non-photometric conditions there). The same result is possible from space with a single small telescope in an orbit with a large continuous viewing zone (CVZ). There are enough bright targets that even telescopes of apertures 15-30 cm can achieve sufficient Poisson statistics to detect $\mu \mathrm{mag}$ oscillations given a sufficiently long time baseline.

MOST (Matthews et al. 1999) is one of three planned space asteroseismology missions; the others being the predominantly French COROT (Michel et al. 2000) and the Danish MONS (Kjeldsen, Bedding, \& Christensen-Dalsgaard 2000). All three are small optical telescopes feeding CCD cameras, but each has 


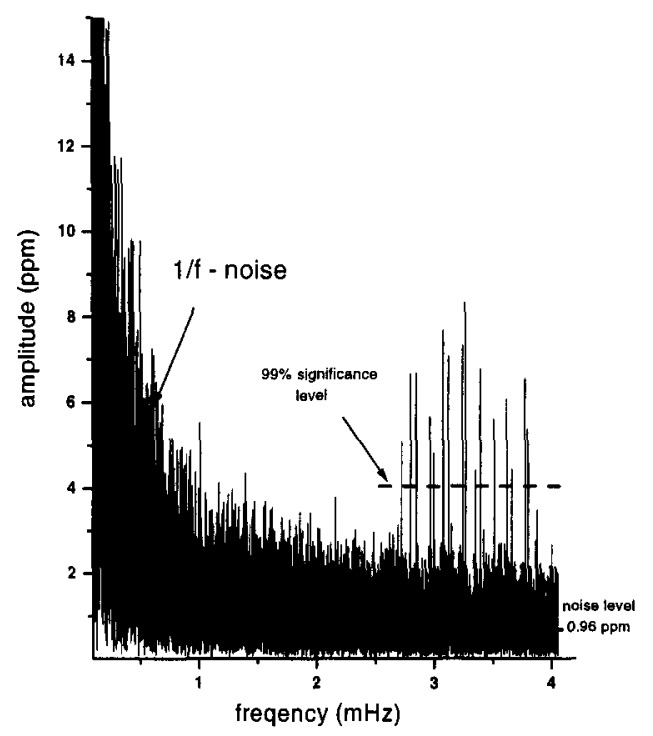

Figure 1. Simulated Fourier amplitude spectrum of MOST photometry of a $V=3 \mathrm{star}$, containing solar-like p-mode oscillations.

adopted a different approach to ultraprecise photometry. MOST is the smallest payload, but exploits new attitude control technology to achieve telescope pointing of \pm 10 arcsec. To minimise the effects of pixel-to-pixel sensitivity variations and CCD degradation in orbit, the MOST telescope will spread the light of a single target star into a large fixed pupil image using Fabry micro-optics.

Simulations of the MOST photometric performance incorporate many random and systematic noise sources in the instrument and the star (including the dominant intrinsic $\sim 1 / \mathrm{f}$ granulation noise) and a duty cycle which accounts for lost data due to enhanced radiation flux in the South Atlantic Anomaly encountered in MOST's planned $800-\mathrm{km}$ polar orbit. Fig. 1 shows that the instrument should be able to detect easily a solar-type eigenspectrum in a bright star after about $10 \mathrm{~d}$ of near-continuous monitoring.

\section{References}

Michel, E., Baglin, A., Barge, P., et al. 2000, in these proceedings, p. 69

Kjeldsen, H., Bedding, T. R., \& Christensen-Dalsgaard, J. 2000, in these proceedings, p. 73

Matthews, J. M., Kuschnig, R., Walker, G. A. H., et al. 1999, http://www. astro.ubc.ca/MOST/ 